\title{
Agreement between rebound (Icare ic200) and applanation tonometry (Perkins) in patients with primary congenital glaucoma
}

\author{
Laura Morales-Fernandez, ${ }^{1,2}$ (D) Pilar Pérez-García, ${ }^{1}$ (D) Federico Saenz-Frances, ${ }^{1}$ \\ Mercedes Molero-Senosiain, ${ }^{1}$ (D) Sofia Garcia-Saenz, ${ }^{1}$ Carmen Dora Mendez, ${ }^{1}$ (D) \\ Enrique Santos Bueso, ${ }^{1}$ Julian Garcia-Feijoo ${ }^{1,3,4}$ and Jose M. Martinez-de-la-Casa ${ }^{1}$ \\ ${ }^{1}$ Ophthalmology Unit, Hospital Clinico San Carlos, Department of Ophthalmology and ORL, Faculty of Medicine, University \\ Complutense (Madrid), Institute "Investigacion Sanitaria del Hospital Clinico San Carlos" (IdISSC), Madrid, Spain \\ ${ }^{2}$ Hospital Universitario Quiron Pozuelo, Madrid, Spain \\ ${ }^{3}$ Institute "Investigaciones Oftalmologicas Ramon Castroviejo", University Complutense, Madrid, Spain \\ ${ }^{4}$ Cooperative Research Network on Age-Related Ocular Disease, and Visual and Life Quality, Instituto de Salud Carlos III, Madrid, Spain
}

\begin{abstract}
.
Purpose: To examine agreement between intraocular pressure (IOP) measurements made using the rebound tonometer Icare ic200 (RT200) and the Perkins handheld applanation tonometer (PAT) in patients with primary congenital glaucoma (PCG). The impacts of several covariables on measurements using the two devices were also assessed.

Materials and Methods: Intraocular pressure measurements were made in a single session in 86 eyes of 86 patients with PCG (46 under anaesthesia, 40 in the office). The order was RT200 then PAT. The variables age, central corneal thickness (CCT), corneal state and anaesthesia were recorded in each patient. Data were compared by determining interclass correlation coefficients (ICC) for each tonometer and representing the differences detected as Bland-Altman plots. Effects of covariables were assessed through univariate and multivariate regression.

Results: Mean IOP difference between tonometers (RT200 minus PAT) was $1.26 \mathrm{mmHg}$ (95\%: 0.22-2.31). Absolute agreement (ICC) was 0.73 (95\% CI: 0.62-0.82). Lower and upper limits of agreement $(95 \%)$ were $-8.06 \mathrm{mmHg}$ (95\% CI: -9.87 to -6.25$)$ and $10.59 \mathrm{mmHg}(95 \%$ CI: 8.77-12.40), respectively. The tonometers showed systematic differences $(a=-4.63 \mathrm{mmHg} ; 95 \%$ CI: -9.11 to -1.44$)$ and proportional differences; for each $\mathrm{mmHg}$ increase in PAT-IOP, the RT200 reading increased by $1.28 \mathrm{mmHg}(b=1.28 ; 95 \% \mathrm{CI}$ : 1.12-1.53). None of the variables tested as predictors were able to explain differences between the tonometers.

Conclusions: Despite the good overall agreement between both tonometers, caution should be taken in high values of IOP, considering the interchangeability of its readings as systematic and proportional differences appear to exist between both methods.
\end{abstract}

Key words: Icare ic200 - primary congenital glaucoma - rebound tonometer - RT200

Acta Ophthalmol.

๑ 2020 Acta Ophthalmologica Scandinavica Foundation. Published by John Wiley \& Sons Ltd

doi: $10.1111 /$ aos. 14701

\section{Introduction}

An elevated intraocular pressure (IOP) is the main modifiable risk factor for glaucoma. While Goldmann applanation tonometry is still the reference standard for pressure measurements, new tonometers based on different working principles are gradually being introduced into routine clinical practice (Arribas-Pardo, Mendez-Hernández, Valls-Ferran, \& Puertas-Bordallo, 2018). For example, the rebound tonometer by iCare (Icare, Tiolat Oy, Helsinki, Finland) has a probe that minimally makes contact with the corneal surface and provides a pressure measurement calculated according to the probe's deceleration as it bounces off the cornea. Rebound tonometry offers a series of advantages over the standard Goldmann technique (Borrego Sanz et al., 2016) including no anaesthesia required, a disposable probe, minimal surface contact and rapid readings. The newer rebound tonometers (iCare Pro) have a magnetic probe that enables measurements with the patient in a lying position. These features are especially useful when the patient is anaesthetized such as when taking measurements in young children or poorly cooperative patients. Since the first iCare TA01m version, new versions have emerged designed to improve both measurement accuracy 
and reliability (Molero-Senosiaín et al., 2019). The latest iCare model ic 200 (RT200), besides having a magnetic probe, has a light to indicate the probe's correct (green) or incorrect (red) position. The device takes six IOP measurements and displays the mean reading on its screen. To ensure the accuracy and reproducibility of measures, the device also has a central corneal probe detector.

Despite all these improvements, to date no study has tested the efficacy of rebound tonometry in children with primary congenital glaucoma (PCG). The present study was therefore designed to assess agreement between IOP measurements made using the RT200 rebound tonometer and handheld Perkins applanation tonometer (PAT) in patients diagnosed with PCG. We also examined whether differences in IOP measurements between the two approaches could be influenced by several covariables including central corneal thickness (CCT), the presence of Haab's striae and the conditions under which measurements were obtained (under general anaesthesia or in an outpatient setting).

\section{Materials and Methods}

This cross-sectional study was designed to assess agreement between RT200 and PAT in a series of patients with congenital glaucoma. Participants were 86 patients diagnosed with unilateral or bilateral PCG recruited from the Glaucoma Dept. of the Hospital Clínico San Carlos (HCSC, Madrid, Spain). These patients met the definition of PCG based on the standardized criteria of the Childhood Glaucoma Research Network guidelines (Beck, Chang, \& Freedman, 2013): an IOP > $22 \mathrm{mmHg}$ on diagnosis, together with clinical evidence of glaucoma such as enlarged corneal diameter, Haab's striae and a glaucomatous appearance of the optic disc head. Patients with other types of early-onset childhood glaucoma (inflammatory, aniridia, dysgenesis or iridocorneal endothelial syndromes) were excluded, as were those who had undergone corneal surgery such as keratoplasty. Common anti-glaucoma procedures in children, such as goniotomy, as the procedure of choice in eyes with corneal transparency, or trabeculectomy, indicated when there is significant corneal oedema or prior goniotomies, were not exclusion criteria.

The study protocol adhered to the tenets of the Declaration of Helsinki and received approval from our hospital Review Board. Written informed consent was obtained from all participants aged 18 years or older or from the parents or legal guardians of participants younger than this age.

The variables compiled for each participant were as follows: age, sex and eye examined (left or right). Only one eye per patient was selected for inclusion. When both eyes were eligible, one eye was randomly selected using a randomization list obtained from www.randomization.com. The ophthalmologic examination included IOP measurement, corneal state, corneal central thickness (CCT), fundus examination and visual acuity.

Using both tonometers, IOP measurements were prospectively obtained in a single session by the same trained examiners (LMF, JGF, CDM) with the iCare ic200 rebound tonometer and a handheld Goldmann applanation tonometer (Perkins; Clement-Clarke, Columbus, OH, USA). The order of device use was RT200 first to avoid corneal deformation after applanation. All measurements were made between 9 am and $1 \mathrm{pm}$ to minimize the effects of diurnal variations. Multiple readings were taken using RT200, and only when RT200 offers a reliable average of IOP after measuring 6 readings, it was recorded (Green light). This version of rebound tonometry offers an indicator of quality obtained after six consecutive measurements. If the 6 readings present an acceptable variation, the device provides a green indication, whereas this signal is yellow or it shows a message of 'repeat' depending on whether that variation is borderline or bad, respectively.

The data collected for corneal state were corneal transparency, Haab's striae, corneal oedema and leukomas. In addition, CCT was measured by ultrasound pachymetry (Dicon P55; Paradigm Medical Industries Inc., Salt Lake City, UT, USA) and expressed as the mean of 5 consecutive measurements obtained in an automated procedure. When possible, we also measured cup-to-disc ratio and best-corrected visual acuity (BCVA) using the Snellen scale (decimal notation).
Patients who cooperated were examined in our office, while young children or those unable to collaborate were examined in the operating room and subjected to IOP measurement as soon as they were sufficiently anaesthetized. In these patients, IOP measurements were always taken first as it has been argued that pressure measurements made immediately after anaesthesia induction are relatively unaffected by anaesthetics (Blumberg et al., 2007). Anaesthesia was induced by introducing $8 \%$ sevoflurane in $100 \%$ oxygen carrier gas and maintained under spontaneous ventilation at a 1 to 2 minimal alveolar concentration of sevoflurane ( $2 \%$ to $4 \%$ ) in $100 \%$ oxygen.

The group of patients examined under anaesthesia were analysed in the supine position and those included in the outpatient clinics group were in upright position.

\section{Statistical analysis}

Overall agreement between the two tonometry methods was assessed through the intraclass correlation coefficient (ICC; random effects, absolute agreement). The Bland-Altman method was used to graphically depict the mean difference between methods, the $95 \%$ confidence interval and the $95 \%$ limits of agreement. PassingBablok regression was used to establish whether systematic and/or proportional differences existed between both tonometers.

To determine whether the differences between RT200 and PAT measurements were conditioned by central corneal thickness, the presence (or not) of Haab's striae, oedema or leukoma, anaesthesia and age, several univariate linear regression models were constructed. In all these models, the dependant variable was the difference between RT200 and PAT measurements, whereas in each, respectively, the predictive variable was each of the aforementioned covariables. Finally, a multivariate model was constructed including as predictive those variables proving significant in the univariate models.

\section{RESULTS}

The study sample comprised 86 eyes of 86 patients with PCG. The baseline demographics of the study groups are 
provided in Table 1. Those cases with poor cooperation were examined under anaesthesia so, as expected, statistical differences were found in age between groups.

Absolute agreement, or ICC, between RT200 and PAT was 0.73 (95\% CI: 0.62-0.82). According to the Bland-Altman plot (Fig. 1), the mean difference between both tonometers (RT200 minus PAT) was $1.26 \mathrm{mmHg}$ (95\%: 0.22 to 2.31$)(p=0.022)$. In $59.31 \%$ of cases, the mean difference was $<3 \mathrm{mmHg}$. The $95 \%$ lower limit of agreement was $-8.06 \mathrm{mmHg}(95 \%$ CI: -9.87 to -6.25$)$, and the $95 \%$ upper limit was $10.59 \mathrm{mmHg}(95 \% \mathrm{CI}$ : 8.77-12.40). As may be seen in the plot, 3 observations were, respectively, below and above the limits of agreement $(3.66 \%$ of the observations, respectively). Differences between RT200 and PAT showed a normal distribution (Fig. 2) (p value of Shapiro-Wilk test $=0.07$ ).

The Passing-Bablok regression line $\left(y=a+b^{*} x\right)$ was constructed according to the following scheme: $R T 200=a+b^{*} P A T$. This analysis identified both systematic differences between both tonometers $(a=-4.63 \mathrm{mmHg} ; 95 \% \mathrm{CI}:-9.11$ to $-1.44)$ and proportional differences such that each $\mathrm{mmHg}$ increase in PAT-IOP was associated with a $1.28 \mathrm{mmHg}$ increase in RT200-IOP $(b=1.28 ; 95 \%$ CI: $1.12-1.53)$. The coefficient of determination between RT200 and PAT was $R^{2}=60.84 \%$. Passing-Bablok analysis is depicted in Fig. 3.

Table 2 provides the data obtained in the univariate linear regression models constructed to explain the extent to which PAT versus RT200 differences could be explained by each of several covariables. As can be inferred from the table, none of these predictors was able to significantly explain the differences between both tonometers. Further, a multivariate linear regression model (Table 3), including all the aforementioned predictors, also failed to explain the variation in differences between the RT200 and PAT measurements.

\section{Discussion}

Accurate IOP measurements are essential for the management of patients with PCG (Baskett, Goen, \& Terry, 1986). When used to take readings in young or uncooperative patients under anaesthesia, the handheld Perkins applanation tonometer is still considered as a reference device. However, the literature confirms reliable and repeatable measurements using rebound devices prior to RT200 in patients with PCG (Martinez-de-la-Casa et al., 2009; Borrego Sanz et al., 2016; Arribas-Pardo et al., 2018).

In the present study, we observed good agreement between RT200 and PAT-IOP measurements in patients with PCG $(\mathrm{ICC}=0.74)$. The mean difference between the readings of both tonometers (RT200 minus PAT) was $1.26 \mathrm{mmHg}$.

Table 1. Demographic and ocular characteristics of the study participants

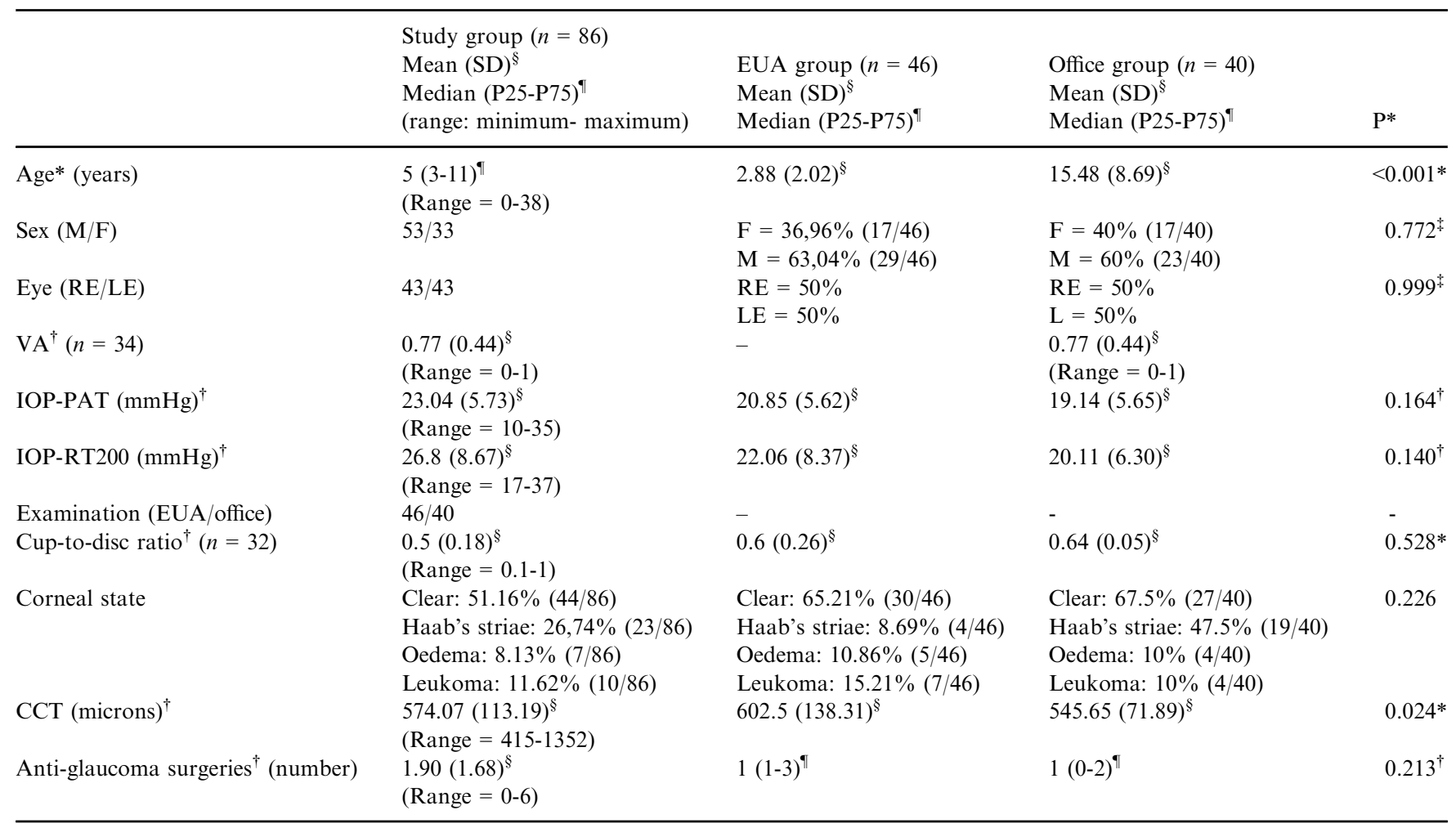

$\mathrm{CCT}=$ central corneal thickness; EUA = examination under anaesthesia; $\mathrm{F}=$ female; $\mathrm{LE}=$ left eye; $\mathrm{M}=\mathrm{male} ; \mathrm{PAT}=\mathrm{Perkins}$ handheld applanation tonometer; RE = right eye; RT200 = iCare 200 rebound tonometer; $\mathrm{SD}=$ standard deviation; VA = visual acuity.

* P T student.

${ }^{\dagger} \mathrm{P}$ Test de Mann-Whitney.

* $\mathrm{P}$ Test chi-squared.

$\S$ Mean (SD).

Median (P25-P75). 


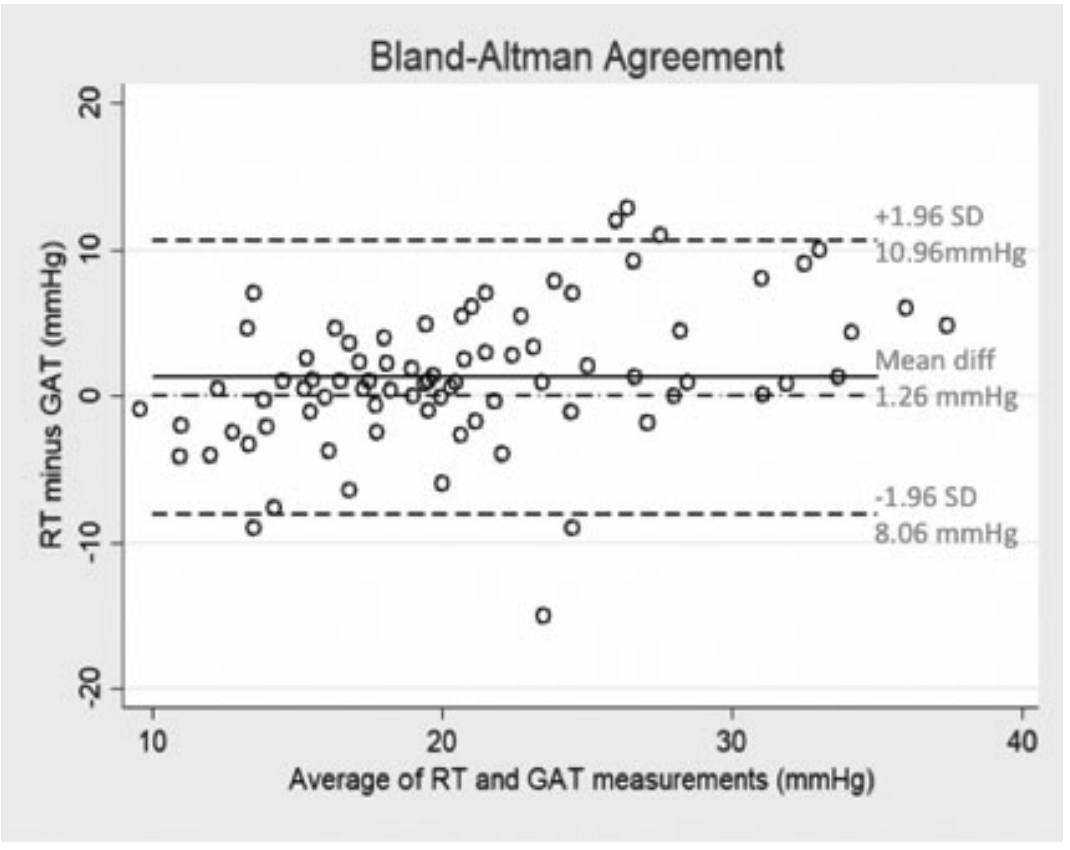

Fig. 1. Bland-Altman plot of RT200 versus PAT.

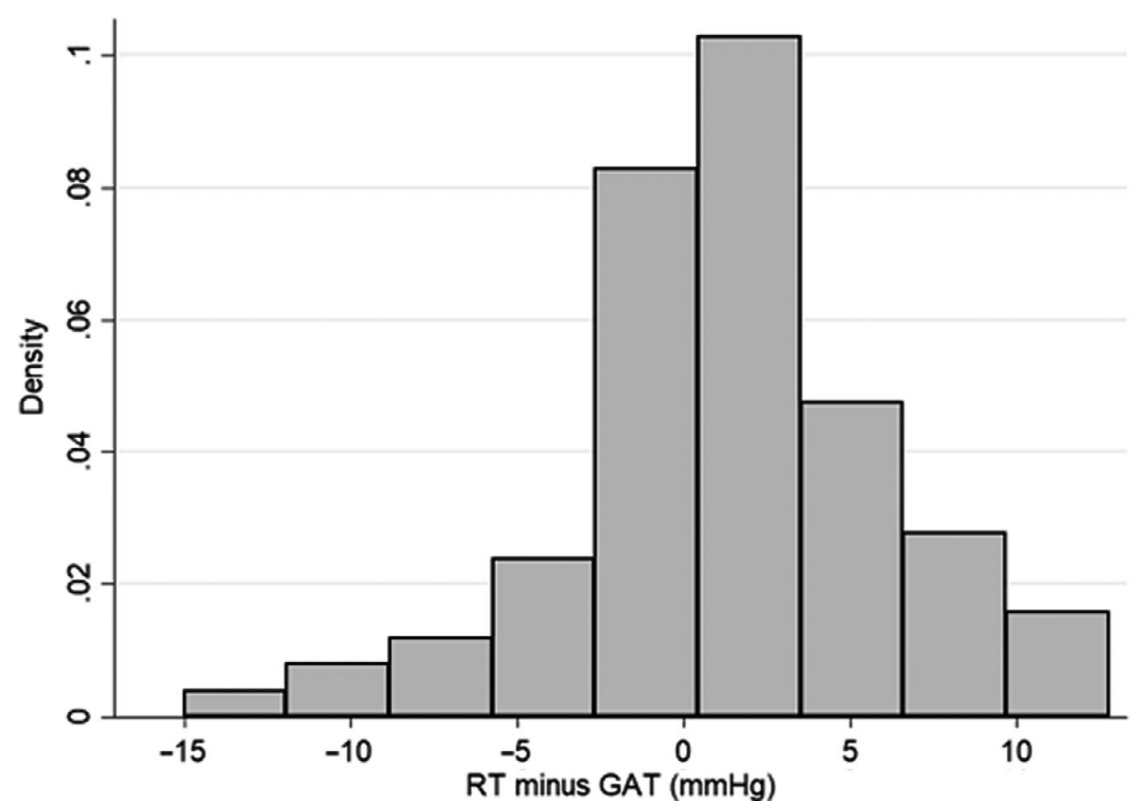

Fig. 2. Distribution of differences between two means: rebound tonometer measurements (RT) minus Perkins measurements (GAT).

The first rebound tonometer version TA01 showed good agreement with PAT in PCG patients and a tendency to overestimate pressures when used in the office (Martinez-de-la-Casa et al., 2009). In healthy children, the mean difference between the two devices was improved, $-0.79 \pm 2.83$ in PCG versus $-0.52 \pm 2.5 \mathrm{mmHg}$ in healthy patients (Esmael, Ismail, Elhusseiny, Fayed, \& Elhilali, 2019). This was devices 0.26) (Arribas-Pardo et al., 2018). The previous version to RT200 was iCare ic100, and while no study has examined its efficacy in PCG, in adults with glaucoma it has shown good agreement with Goldmann applanation tonometry (ICC $=0.93)(9)$.

However, despite the good correlation $(0.73)$ and the small mean difference between the readings of the two tonometers $(1.26 \mathrm{mmHg})$, the BlandAltman plot indicates that the $95 \%$ limits of agreements are rather wide ranging from $-8.06 \mathrm{mmHg}$ to $10.59 \mathrm{mmHg}$. In this regard, is worth noting that in $40 \%$ of the eyes examined, the differences observed between IOP readings between RT200 and PAT were higher than $3 \mathrm{mmHg}$.

The linear regression models revealed that none of the covariates analysed affected the differences observed between PAT and RT200 readings. Furthermore, systematic and proportional differences were detected (Passing-Bablok regression equation: RT200-IOP $=-4.63+1.28 \quad$ PATIOP). As we could observe in Table 2, higher values of PAT-IOP result on higher readings of RT200-IOP when PAT-IOP is higher than $17 \mathrm{mmHg}$; in contrast, RT200-IOP would be predicted to be lower than PAT-IOP if the PAT-IOP is lower than $16 \mathrm{mmHg}$. This phenomenon could explain the tendency of infra or overestimation of iCare tonometry when compared with Goldmann tonometry.

Authors such as Molero-Senosiaín et al. (2019) reported significant underestimation of IOP when using the iCare 100 tonometer versus Icare PRO and Perkins. Nakakura et al. (Nakakura et al., 2019) also recorded lower IOPs with iCare 100 compared to iCare TA01 and GAT. In contrast, other authors argued that rebound tonometry (iCare TA01 and Icare Pro) leads to significantly higher IOP values $(1.8 \mathrm{mmHg})$ than GAT (Martinez-dela-Casa et al., 2005; Martinez-de-laCasa et al., 2006).

In addition, controversy exists over the possible influence of CCT in the differences observed between rebound and applanation tonometry. While some studies have ruled out any effects of CCT on Icare PRO measures (Nakakura et al., 2015), others have reported higher IOP measurements with increasing CCT (Brusini, Salvetat, Zeppieri, Tosoni, \& Parisi, 2006). In a 


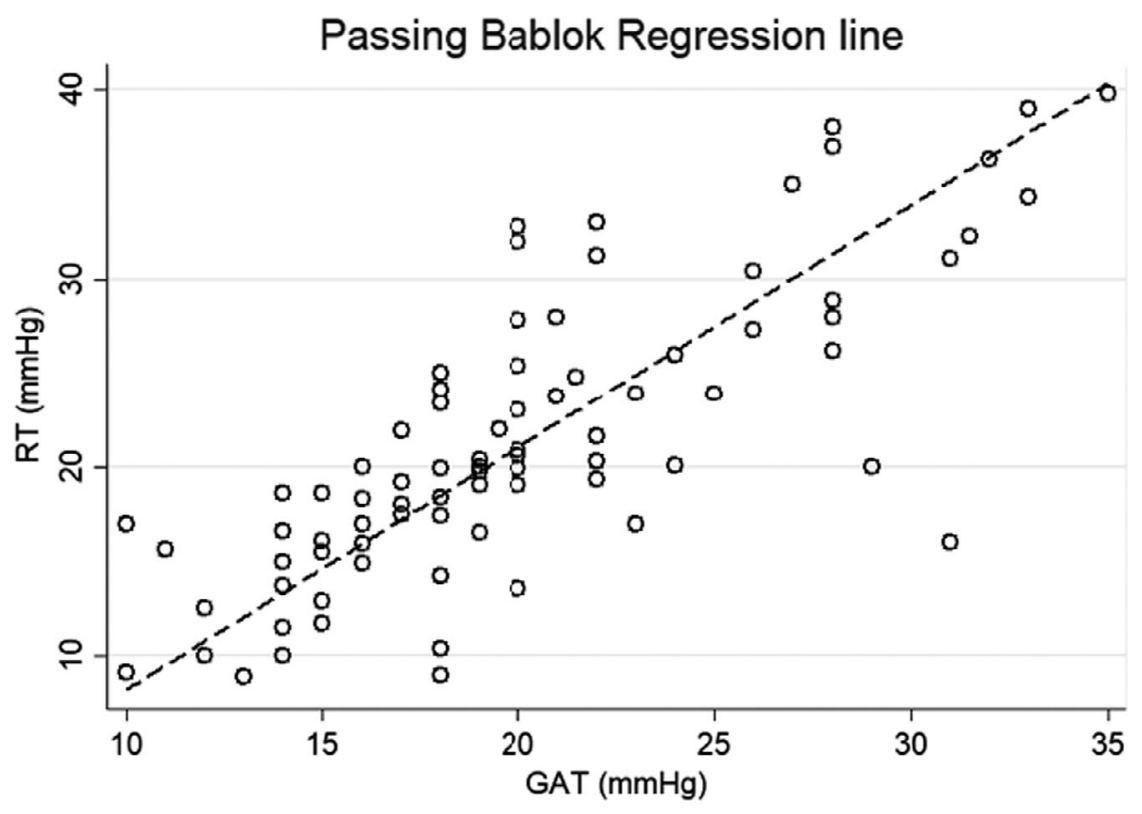

Fig. 3. Passing-Bablok regression analysis.

Table 2. Univariate linear regression models depicting the relationship between RT200 versus PAT measurement differences and predictors. Note that each row represents one linear regression model

\begin{tabular}{|c|c|c|c|c|c|c|c|c|}
\hline Predictors & Slope* & $\mathrm{P}$ & $95 \% \mathrm{CI}$ & & Slope $^{\dagger}$ & $\mathrm{P}$ & $95 \% \mathrm{CI}$ & \\
\hline CCT & 0.00 & 0.53 & -0.01 & 0.01 & 0.00 & 0.43 & -0.01 & 0.01 \\
\hline EUA vs office & -0.85 & 0.43 & -2.95 & 1.26 & 0.57 & 0.70 & -2.39 & 3.54 \\
\hline Age & -0.09 & 0.13 & -0.22 & 0.03 & - & & & \\
\hline Presence of Haab's striae & 0.70 & 0.61 & -1.58 & 2.98 & 0.11 & 0.93 & -2.36 & 2.57 \\
\hline Oedema & -0.30 & 0.873 & -4.07 & 3.46 & -0.46 & 0.823 & -4.57 & 3.64 \\
\hline Leukoma & -0.72 & 0.642 & -3.80 & 2.36 & -0.82 & 0.601 & -3.93 & 2.29 \\
\hline
\end{tabular}

$\mathrm{CCT}=$ central corneal thickness; EUA = examination under anaesthesia.

* Unadjusted.

${ }^{\dagger}$ Adjusted for age and gender.

Table 3. Covariables entered in the multivariate linear regression model of the relationship between RT200 and PAT measurement differences and predictors

\begin{tabular}{lrlll}
\hline Predictor & Slope & P & $95 \%$ CI & \\
\hline CCT & 0.00 & 0.34 & -0.01 & 0.00 \\
Presence of Haab's striae & 0.75 & 0.57 & -1.88 & 3.37 \\
EUA vs office & -0.40 & 0.81 & -3.69 & 2.89 \\
Age & -0.07 & 0.41 & -0.24 & 0.10 \\
Oedema & -0.46 & 0.81 & -4.39 & 3.46 \\
Leukoma & 0.63 & 0.72 & -2.93 & 4.18 \\
\hline
\end{tabular}

$\mathrm{CCT}=$ central corneal thickness; EUA $=$ examination under anaesthesia.

recent report, it was described that IOPs measured using iCare 100 and iCare TA01 were moderately but significantly affected by CCT (Nakakura et al., 2019), unlike our observations for RT200. These variations among the different studies could be attributable to the varying probe lengths of the different iCare devices or the fact that additional corneal features may play a role, such as corneal hysteresis and the corneal resistance factor, which are clearly modified in PCG patients (Perucho-González et al., 2017; Brown et al., 2018).

Another important factor to consider in this paediatric glaucoma population is the effect of corneal changes induced by hydrops or scarring. Here, we detected no effects of corneal state on our rebound tonometry readings. This finding is consistent with observations by Tal et al. (Badakere et al., 2019) who reported agreement between Icare PRO and Perkins in scarred corneas similar to clear corneas in PCG. These last authors, nevertheless, highlighted a trend towards overestimation of IOP values higher than $19 \mathrm{mmHg}$ both in both clear and scarred corneas.

Intraocular pressure measurements may also be affected by a supine decubitus measurement position and anaesthesia. We did not detect any influence of supine decubitus position (EUA group) in our study; however, the effect of anaesthesia could interfere with our results. We should mention an increased use of rebound tonometry in children given it is a quick, simple method that does not require anaesthetic eye drops. In effect, this tool could help reduce the number of examinations under anaesthesia in children 
with PCG requiring periodic examinations (Grigorian et al., 2015). Also, while in our study age was neither found to affect differences in IOP readings between devices, others have warned that in very young patients, rebound tonometry tends to overestimate office IOP measurements by as much as $>8 \mathrm{mmHg}$ (Chan et al., 2015). It has been observed that iCare tonometry is highly sensitive to breath holding in the clinical practice so it is not unusual to take multiple readings of IOP especially in children. This can be assumed as a limitation; however, in our study, multiple readings were taken, and only when RT200 offers a reliable average of IOP after measuring 6 readings, it was recorded (green light).

The main limitation of our study was patient selection bias. The reason for this is that patients with PCG are difficult to recruit as this disease is not very prevalent, and our institution is a national reference centre that manages patients in whom IOP control is poor, and the disease course is complicated. Another limitation is that the iCare ic200 used here, despite providing more accurate pressure readings, can only be used on the central cornea and not the peripheral cornea or limbus (Bontzos et al., 2017). In young children, this makes it difficult to measure IOP.

Other limitation is that three examiners participated in the study due to the low prevalence of this disease and the difficulty for recruiting cases. Interexaminer variation could interfere to the results. On the other hand, other variables could interfere in the RT200 readings, for example biomechanical properties or anti-glaucoma medications and surgeries. Further studies are required to address these factors.

As a conclusion, despite the good overall agreement between both tonometers, caution should be taken when considering the interchangeability of its reading as systematic and proportional differences appear to exist between both methods.

\section{References}

Arribas-Pardo P, Mendez-Hernández C, VallsFerran I \& Puertas-Bordallo D (2018):
Icare-Pro rebound tonometer versus handheld applanation tonometer for pediatric screening. J Pediatr Ophthalmol Strabismus 55: 382-386.

Badakere SV, Rao HL, Ali MH, Mandal AK, Choudhari NS, Chandrasekhar G \& Senthil S (2019): Comparison of rebound tonometry and handheld applanation tonometry in pediatric glaucoma with clear and scarred corneas. Ophthalmology 126: 1330-1332.

Baskett JS, Goen TM \& Terry JE (1986): A comparison of Perkins and Goldmann applanation tonometry. J Am Optom Assoc 57: 832-834.

Beck A, Chang TC \& Freedman S (2013): Definition, classification, differential diagnosis. In: Weinreb RN, Grajewski A, Papadopoulos M, Grigg J \& Freedman S (eds). Childhood Glaucoma. Amsterdam: Kugler Publications, 3-10.

Blumberg D, Congdon N, Jampel H, Gilbert D, Elliott R, Rivers R, Munoz B \& Quigley $H$ (2007): The effects of sevoflurane and ketamine on intraocular pressure in children during examination under anesthesia. Am J Ophthalmol 143: 494499.

Bontzos G, Agiorgiotakis M, Kapsala Z \& Detorakis E (2017): Limbal rebound tonometry: clinical comparisons and applications. Graefe's Arch Clin Exp Ophthalmol 255 : 1795-1799.

Borrego Sanz L, Morales-Fernandez L, Martínez de-la-Casa JM, Sáenz-Francés F, Fuentes M \& García-Feijóo J (2016): The Icare-Pro rebound tonometer versus the hand-held applanation tonometer in congenital glaucoma. J Glaucoma 25: 149-154.

Brown L, Foulsham W, Pronin S \& Tatham AJ (2018): The influence of corneal biomechanical properties on intraocular pressure measurements using a rebound self-tonometer. J Glaucoma 27: 511-518.

Brusini P, Salvetat ML, Zeppieri M, Tosoni C \& Parisi L (2006): Comparison of ICare tonometer with Goldmann applanation tonometer in glaucoma patients. J Glaucoma 15: 213-217.

Chan WH, Lloyd IC, Symes RJ, Ashworth JL, Cosgrove E, Pilling R \& Biswas S (2015): Accuracy of intraocular pressure measurement with the Icare tonometer in children . Asia-Pacific J Ophthalmol (Philadelphia, Pa.) 4: 357-359.

Esmael A, Ismail YM, Elhusseiny AM, Fayed AE \& Elhilali HM (2019): Agreement profiles for rebound and applanation tonometry in normal and glaucomatous children. Eur $\mathbf{J}$ Ophthalmol 29: 379-385.

Grigorian F, Grigorian AP, Li A, Sattar A, Krishna R \& Olitsky SE (2015): Comparison of the Icare rebound tonometry with the Goldmann applanation tonometry in a pediatric population. J AAPOS 19: 572-574.

Martinez-de-la-Casa JM, Garcia-Feijoo J, Castillo A \& Garcia-Sanchez J (2005): Reproducibility and clinical evaluation of rebound tonometry. Invest Ophthalmol Vis Sci 46: 4578-4580.

Martinez-de-la-Casa JM, Garcia-Feijoo J Saenz-Frances F, Vizzeri G, FernandezVidal A, Mendez-Hernandez C \& GarciaSanchez J (2009): Comparison of rebound tonometer and Goldmann handheld applanation tonometer in congenital glaucoma. $\mathbf{J}$ Glaucoma 18: 49-52.

Martinez-de-la-Casa JM, Garcia-Feijoo J, Vico E, Fernandez-Vidal A, Benitez del Castillo JM, Wasfi M \& Garcia-Sanchez J (2006): Effect of corneal thickness on dynamic contour, rebound, and goldmann tonometry. Ophthalmology 113: 2156-2162. Molero-Senosiaín M, Morales-Fernández L, Saenz-Francés F, García-Feijoo J \& Martínez-de-la-Casa JM (2019). Analysis of reproducibility, evaluation, and preference of the new iC100 rebound tonometer versus iCare PRO and Perkins portable applanation tonometry. Eur J Ophthalmol, 1120672119878017. Advance online publication. doi: 10.1177/1120672119878017.

Nakakura S, Mori E, Fujio Y et al. (2019): Comparison of the intraocular pressure measured using the new rebound tonometer Icare ic100 and Icare TA01i or Goldmann Applanation Tonometer. J Glaucoma 28: 172-177.

Nakakura S, Mori E, Yamamoto M, Tsushima Y, Tabuchi H \& Kiuchi Y (2015): Intradevice and interdevice agreement between a rebound tonometer, Icare PRO, and the Tonopen XL and Kowa hand-held applanation tonometer when used in the sitting and supine position. J Glaucoma 24: 515-521.

Perucho-González L, Sáenz-Francés F, Morales-Fernández L, Martínez-de-la-Casa JM, Méndez-Hernández CD, Santos-Bueso E, Brookes JL \& García-Feijoó J (2017): Structural and biomechanical corneal differences between patients suffering from primary congenital glaucoma and healthy volunteers. Acta Ophthalmol 95: e107-e112.

Received on July 14th, 2020.

Accepted on November 9th, 2020.

Correspondence:

Laura Morales-Fernandez, MD, PhD

Ophthalmology Unit

Hospital Clinico San Carlos

Instituto de Investigacion Sanitaria del

Hospital Clinico San Carlos (IdISSC)

Professor Martin Lago SN

Madrid

Spain

Tel: +34666 314403

Fax: +34913 303132

E-mail: lauramoralesfernandez@gmail.com 